16 | 2013

NOVECENTO... E DINTORNI

« On ne naît pas... on le devient »

\title{
La notion de genre en terre catholique. Le dispositif patriarcal de subjectivation genrée dans la réflexion critique de Luisa Accati
}

Anna Mirabella

\section{OpenEdition}

\section{Journals}

Édition électronique

URL : http://journals.openedition.org/cei/1100

DOI : $10.4000 /$ cei. 1100

ISSN : 2260-779X

Éditeur

UGA Éditions/Université Grenoble Alpes

\section{Édition imprimée}

Date de publication : 30 juin 2013

Pagination : 65-78

ISBN : 978-2-84310-245-5

ISSN : 1770-9571

Référence électronique

Anna Mirabella, « La notion de genre en terre catholique.

Le dispositif patriarcal de subjectivation genrée dans la réflexion critique de Luisa Accati », Cahiers d'études italiennes [En ligne], 16 | 2013, mis en ligne le 15 décembre 2014, consulté le 26 mars 2021 URL : http://journals.openedition.org/cei/1100 ; DOI : https://doi.org/10.4000/cei.1100 


\title{
LA NOTION DE GENRE EN TERRE CATHOLIQUE. LE DISPOSITIF PATRIARCAL DE SUBJECTIVATION GENRÉE DANS LA RÉFLEXION CRITIQUE DE LUISA ACCATI
}

\author{
Anna Mirabella \\ Université de Nantes
}

Le sexe biologique est-il une construction culturelle au même titre que le genre? La "matérialité» du corps et le sexe naturel sont-ils des notions produites par une certaine configuration du genre, définie dans le cadre du dispositif patriarcal de normalisation? Cette idée est à la base de la réflexion de Judith Butler qui considère comme nécessaire l'élimination de toute référence au sexe biologique dans la définition du genre. Selon Butler, la conception binaire du sexe naturel n'est qu'un postulat normatif de la culture patriarcale qui imposerait le principe de l'hétérosexualité normative obligatoire.

Le genre, c'est l'ensemble des moyens discursifs/culturels par quoi la «nature sexuée» ou un "sexe naturel» est produit et établi dans un domaine "prédiscursif», qui précède la culture, telle une surface politiquement neutre sur laquelle intervient la culture après coup. [...] Dire que le sexe est produit en tant que donnée prédiscursive, c'est dire que cette production est un effet de cet appareil de construction culturelle qu'est le genre ${ }^{\mathrm{I}}$.

Le maintien de la distinction sexe naturel/genre n'est qu'une «fiction régulatrice qui consolide et naturalise les régimes convergeant de pouvoirs liés à la domination masculine et l'hétérosexisme ${ }^{2}$ ». Il est l'effet de pouvoir d'une "hétérosexualité obligatoire et naturalisée» produisant la différenciation binaire entre masculin et féminin ainsi que le désir hétérosexuel.

I. Judith Butler, Trouble dans le genre (Gender Trouble). Le féminisme et la subversion de l'identité, Paris, La Découverte, 2006 (éd. originale 1990), p. 69.

2. Ibid., p. IIо. 
Ce dernier serait l'élément décisif qui permet de «stabiliser la métaphysique de la substance qui le sous-tend ${ }^{3}$ ».

Donc, pour affranchir les sujets de l'ordre symbolique patriarcal imposant l'hétérosexualité comme obligation normative, il faut effacer les marques des genres normalisés inscrites sur la surface des corps et présentées comme les qualités substantielles du corps. Il faut "désubstantialiser» le corps à partir de la déconstruction de sa supposée nature sexuée. "Le sexe», affirme Butler, ne relève pas "d'une facticité anatomique prédiscursive $^{4}$ ». Il relève d'une construction normative fondée sur le principe d'une hétérosexualité obligatoire imposant la reproduction comme "devoir» et reléguant l'homosexualité au statut de déviance. Le sexe n'est pas une "vérité intérieure», résultant de prédispositions biologiques du corps mais un effet de surface, "une signification produite sur un mode performatif (et donc qu'il n'est pas) laquelle, si elle est déliée de son intérieur dénaturalisé et de sa surface, peut devenir l'occasion d'une prolifération parodique et d'un jeu subversif sur les significations genrées'».

D’où vient cette construction normative du genre hétéronormé et qui impose la fiction d'un sexe naturel? Quelle est l'histoire de ce dispositif de pouvoir qui effacerait la prééminence de l'homosexualité des sujets - que Butler affirme sur la base d'une relecture de la dynamique œedipienne ${ }^{6}$ au profit d'une hétérosexualité obligatoire et naturalisée?

L'idée que le corps n'aurait d'existence signifiante que par la force performative du discours, ne représente-elle un postulat idéaliste reconduisant implicitement une modalité d'appréhension du corps métaphysique ${ }^{7}$ ? Est-il pertinent de postuler la dimension discursive du sexe biologique

3. Ibid., p. 93.

4. Ibid., p. 7I.

5. Ibid., p. IIo. Pour un approfondissement critique sur l'usage de la notion de performativité chez Judith Butler, voir Bruno Ambroise, "Judith Butler et la fabrique discursive du sexe», Raisons politiques, 4, I2, 2003, p. 99-I2I.

6. La différence naturelle entre deux sexes serait en effet le produit d'une "naturalisation" du désir hétérosexuel qui serait le postulat fondamental de la théorie psychanalytique du complexe d'Edipe ainsi que de celle de l'universalité de l'interdit de l'inceste élaborée par Lévi-Strauss. Butler remet en question ces deux théories considérant que les êtres humains seraient marqués par la prééminence du désir homosexuel qui serait ensuite refoulé et substitué par une hétérosexualité imposée comme norme. Sur l'analyse de Judith Butler de la fonction normative de l'interdit de l'inceste voir «Prohibition, psychanalyse et production de la matrice hétérosexuelle", dans Trouble dans le genre, ouvr. cité, p. II3-I77. Pour un approfondissement, voir aussi Judith Butler, "Identification fantasmatique et assomption du sexe", dans Ces corps qui comptent. De la matérialité et des limites discursives $d u$ "sexe", Paris, Éditions Amsterdam, 2009 (éd. originale 1993), p. 294 et Judith Butler, "Le genre de la mélancolie / L'identification refusée», dans La vie psychique du pouvoir, Paris, Éditions Léo Scheer, 2002 (éd. originale 1997), p. 199-222.

7. Cette objection a été formulée maintes fois contre les thèses de Judith Butler. L'auteur y répond, en infléchissant quelque peu sa conception hyper-constructiviste du corps théorisée dans Trouble dans le genre. Voir aussi du même auteur le chapitre "La matière du corps", dans Ces corps qui comptent. De la matérialité et des limites discursives $d u$ "sexe", ouvr. cité, p. 39-68. Pour une réflexion critique sur le constructivisme de 
pour défaire la hiérarchisation des sujets à partir de leur identité sexuée et/ou sexuelle? Dans ce que Butler appelle, de manière bien trop vague, la "culture patriarcale», les identités sexuées auraient été naturalisées pour fonder la construction du sujet hétérosexuel dont la finalité ultime ne serait que la reproduction. La déconstruction de la différence binaire des sexes permettrait-elle de penser autrement le fait de la reproduction et son incidence sur les formes de subjectivation genrées? Faudrait-il exclure la question de la reproduction sexuée des êtres humains du champ des formes de subjectivation?

Judith Butler nie toute pertinence de la question de la reproduction dans la construction du sujet sexué. Dans ses travaux, l'engendrement apparaît simplement comme une imposition normative finalisée à assurer la domination masculine. Ce réductionnisme est hautement problématique tout particulièrement au moment où l'évolution des nouvelles technologies de reproduction ainsi que les incertitudes quant à la redéfinition des rôles paternels et maternels engendrent au sein de l'espace public des interrogations que l'on ne peut pas négliger et qui intéressent un grand nombre de femmes et hommes. Est-on vraiment obligé de choisir entre un discours normatif axé sur l'imposition de l'hétérosexualité obligatoire et naturelle en vue de la reproduction et un discours qui déconstruit cette structure hétéronormée mais qui, en effaçant la dualité des sexes, ne peut plus rendre compte ni contribuer à signifier autrement la différence des sexes et leur complémentarité dans la reproduction biologique? Enlever toute pertinence à la notion de différence des sexes ne risque pas de reconduire le déni de l'une des dimensions qui caractérisent les êtres de sexe féminin — la faculté d'engendrer dans leur propre corps — et d'imposer une nouvelle neutralité normative masculine?

Nous nous proposons de discuter les hypothèses de Butler concernant la structure hétéronormée du patriarcat occidental et sa production du concept normatif de sexe naturel à partir des travaux de l'historienne et ethnologue Luisa Accati.

À la différence de Judith Butler, Luisa Accati n'étudie pas la construction culturelle des genres uniquement à travers leur conceptualisation psychanalytique, anthropologique et philosophique (Aristote et Platon d'un côté et puis de Hegel à Foucault et Derrida de l'autre). À partir de l'étude de l'imaginaire religieux, Luisa Accati reconstruit dans une perspective généalogique et historique l'ordre normatif symbolique qui a organisé la 
définition du genre dans l'Occident chrétien. Notre imaginaire est en effet encore bien imprégné de la culture religieuse dont la symbolique continue d'influencer notre manière de penser la sexualité et la relation entre identité sexuée et reproduction. Ces deux questions ont été en effet au cour de la redéfinition moderne du christianisme selon deux modalités distinctes dans le champ protestant et catholique. La Réforme du Xvi ${ }^{\text {e }}$ siècle, comme le montre Luisa Accati, a produit deux univers symboliques du genre qui ont durablement marqué nos sociétés et dont il faut prendre en compte les spécificités.

Dans cette perspective, Luisa Accati identifie d'une part les différences structurelles entre le patriarcat protestant et catholique. D'autre part, en analysant l'évolution du dogme de la Conception Immaculée, elle souligne deux caractéristiques essentielles de l'imaginaire catholique : la très forte dévalorisation de l'hétérosexualité et la construction d'un imaginaire de la domination ecclésiastique marqué implicitement par une connotation homosexuelle.

Le travail d'historicisation des normes et la reconstruction généalogique des héritages idéologiques d'une culture, comme l'avait bien indiqué Michel Foucault, sont la condition indispensable pour déconstruire les systèmes de domination ${ }^{8}$. De même, seul la prise en compte des spécificités des systèmes symboliques des divers contextes religieux, comme le montre le travail de Luisa Accati, permet une véritable compréhension des enjeux dans la construction du genre dans la pensée occidentale.

\section{La construction des identités genrées dans la symbolique catholique}

\section{Dans l'ouvrage Il Mostro e la bella. Padre e madre nell'educazione cattolica dei sentimenti ${ }^{9}$, Luisa Accati propose une étude généalogique de la sym-}

8. En élaborant la notion de norme, Judith Butler se réfère constamment à Michel Foucault. Cependant, tandis que pour l'auteur la norme est un énoncé dont l'efficacité est expliquée en termes de performativité, pour Foucault la définition et l'efficacité de la norme s'expliquent en fonction du contexte historique de son apparition. Pour Foucault, la norme n'a pas d'efficacité en elle-même en tant qu'énoncé. Celle-ci est déterminée par son usage dans un contexte historique spécifique, dans ce que Foucault appelle un espace stratégique de luttes où les sujets sont appelés à se penser et à se positionner, sans jamais être complètement assujettis au cadre normatif. Pour une analyse critique de la notion de norme, voir Pierre Macherey, De Canguilhem à Foucault, la force des normes, Paris, Fabrica, 2009, p. 145 .

9. Luisa Accati, Il mostro e la bella. Padre e madre nell'educazione cattolica dei sentimenti, Milano, Raffaello Cortina, 1996, p. 292. Pour une synthèse du contenu de l'ouvrage, je me permets de renvoyer à mon compte rendu, Anna Mirabella, "Luisa Accati. Il mostro e la bella. Padre e madre nell'educazione cattolica dei sentimenti ", dans Clio, 26, 2007. Disponible sur <http://clio.revues.org/index7073.html> (consulté le ro février 20I2). 
bolique catholique concernant la construction des rôles familiaux et des identités genrées à travers lesquels l'Église catholique a affermi progressivement son emprise sur le corps politique.

La reformulation de la symbolique catholique commence au XVI ${ }^{\mathrm{e}}$ siècle, dans le cadre du réarrangement des prérogatives de l'autorité temporelle et spirituelle. La Réforme se produit au moment de la naissance de l'État moderne, nouvelle forme de la communauté politique dont l'autorité s'affirme à travers un processus d'émancipation progressive de l'autorité ecclésiastique. La redéfinition des liens entre l'autorité temporelle et spirituelle va se concrétiser selon des modalités spécifiques dans le camp catholique et protestant en fonction de choix doctrinaires profondément différents.

Luisa Accati souligne en particulier l'importance de trois points doctrinaires qui vont marquer en profondeur les cultures dominées par l'une ou l'autre confession : la diverse conception des conséquences de la concupiscence qui amène à deux visions opposées du mariage; une valorisation très spécifique de la figure de la Vierge ainsi qu'un usage différent des images sacrées qui, abolies par les protestants, seront au cœur de la refonte du catholicisme romain. Pour les réformés, le désir et l'acte sexuel, moyen de transmission du pêché originel, demeurent un mal inévitable, lié à la condition humaine. Seul le mariage, rigoureusement finalisé à la reproduction, peut limiter les conséquences négatives de la concupiscence. Ainsi, il est imposé à tous les fidèles, y compris les ecclésiastiques.

Tandis que les protestants abolissent l'obligation du célibat décrétée par la réforme grégorienne, les catholiques maintiennent cette norme et les prêtres n'accèdent pas au mariage. Selon la doctrine de Rome, une catégorie d'hommes - les clercs - ont la faculté de s'affranchir des tentations de la chair et, en vertu de cela, les ecclésiastiques sont considérés comme "moralement supérieurs" par rapport à ceux qui ne savent pas maîtriser le désir sexuel, les laïcs. La supériorité morale des ecclésiastiques légitime leur autorité sur les laïcs, qui ne peuvent s'amender qu'en acceptant l'union conjugale sous le contrôle de l'Église de Rome.

Dans l'imaginaire catholique, comme le souligne Luisa Accati, se définit une hiérarchie nouvelle entre les ecclésiastiques et les laïcs, c'est-à-dire entre les hommes "purs» et les «impurs", qui doivent être gouvernés par les premiers. Dans les pays protestants tous les hommes, clercs et laïcs, sont situés sur le même plan au sein de la sphère publique et leur supériorité sur les femmes se renforce. Le critère normatif qui fonde l'exercice de l'autorité est défini en fonction de la différence de sexes : tous les hommes sont habilités à l'exercice du pouvoir tandis que toutes les femmes en sont 
exclues. Dans le contexte protestant domine une image de l'autorité exclusivement masculine et paternelle. Ici effectivement le modèle normatif du couple hétérosexuel s'impose de manière univoque. L'univers symbolique protestant est patriarcal au sens propre : l'autorité est construite uniquement dans la dimension masculine paternelle comme "pouvoir des pères». Dans l'imaginaire protestant, avec l'élimination du culte des saints et des saintes ainsi qu'avec la redéfinition de la Vierge comme simple mère exemplaire, aucun symbole féminin n'est plus valorisé en tant que figure d'autorité. Cette exclusion d'un modèle normatif féminin d'autorité renforce l'exclusion des femmes de la sphère publique du pouvoir, d'une part. D'autre part, le seul moyen dont les femmes disposent pour se voir reconnue une autorité, c'est de se conformer à un modèle masculin paternel qui devient progressivement la figure unique du sujet d'autorité.

Dans le catholicisme, les hommes laïcs sont obligés de se marier du fait de leur incapacité à résister à la concupiscence. Impurs, ils perdent toute légitimation en tant que figures d'autorité, ce qui demeure la prérogative d'une autre classe d'hommes purs et moralement supérieurs : les clercs ${ }^{10}$. Dans l'ordre symbolique catholique, la reconnaissance de l'autorité dans la sphère publique n'est donc pas fondée sur le critère du sexe naturel. Si aucune femme ne figure comme sujet d'autorité, seulement une classe d'hommes est identifiée en tant que telle : les ecclésiastiques.

Ainsi l'idéologie de la Contre-Réforme pose les bases pour un patriarcat très spécifique qui est moins un "pouvoir des pères» qu'un "pouvoir des fils», un pouvoir des ecclésiastiques qui, liés par le vœu de chasteté, n'accèdent pas à la paternité, demeurant «fils" à jamais.

$\mathrm{Si}$ dans les deux confessions issues de la Réforme les femmes sont également exclues de la sphère publique de l'autorité, leur position de subordination et leur identification sociale sont structurellement différentes, car elles sont définies à l'intérieur de deux systèmes spécifiques de hiérarchisations entre les hommes.

En situant la genèse de l'autorité au sein des relations familiales, dans le sillage de la psychanalyse freudienne, Luisa Accati distingue le contexte protestant où tous les hommes accèdent à la paternité définissant ainsi leur autorité, du contexte catholique où l'autorité revient à ceux qui demeurent exclus de la paternité. Il en dérive une construction de l'autorité radica-

Io. En d'autres termes, dans un contexte où seuls les hommes sont légitimés en tant que figures de pouvoir, le genre masculin apparaît comme le genre du pouvoir. Une femme peut accéder à l'exercice de l'autorité mais en se conformant au modèle masculin qui demeure la condition légitimant l'autorité. Cette caractérisation de la relation entre genre et pouvoir représente, selon Luisa Accati, la principale différence dans la structuration du symbolique et de l'imaginaire collectif entre les pays de tradition catholique et protestante. 
lement différente. Tandis que dans le cadre protestant le modèle axiomatique de l'autorité est la figure divine paternelle, dans le contexte catholique il est représenté par une figure féminine maternelle : la Vierge. Dans la doctrine de la Contre-Réforme, le culte de la Vierge acquiert une importance inédite. Consacrée Mère de l'Église, la Vierge devient le symbole de l'institution de la médiation, la figure symbolique qui incarne l'autorité des ecclésiastiques. Dès lors, comment fonctionne-t-il ce "pouvoir des fils", les prêtres, se donnant comme symbole d'identification une figure maternelle et féminine? Le sens de la sanctification de Marie et sa nouvelle fonction en tant que pivot de la diffusion du dispositif de domination de l'idéologie catholique est analysé par Luisa Accati à travers l'étude de l'évolution de l'iconographie sacrée de Marie qui aboutit à l'élaboration de l'icône de la Vierge Immaculée. La promotion du culte de l'Immaculée sera un élément clé dans la stratégie de diffusion de l'idéologie de l'Église de la Contre-Réforme. La prise en compte de l'iconographie pour expliquer la genèse différentielle de l'imaginaire catholique et protestant est essentielle car, comme on le sait, les réformés abolissent le culte des images tandis que les catholiques en font le fer de lance de leur pénétration dans le corps politique.

\section{L'autorité des ecclésiastiques au prisme de la Conception Immaculée}

Approuvé tardivement par l'Église de Rome en I854 avec la Bulle papale Ineffabilis Deus, le dogme de la Conception Immaculée commence à être élaboré au XII ${ }^{\mathrm{e}}$ siècle au sein de la culture monastique franciscaine. Si l'opposition des dominicains freine son institutionnalisation, à partir de la Contre-Réforme le puissant ordre des jésuites se range du côté des franciscains contribuant efficacement à sa diffusion. Pour l'approbation du dogme il faudra attendre plus de deux siècles, pendant lesquels la représentation de la Conception Immaculée devient un thème central dans l'iconographie catholique. Les plus grands peintres de l'âge classique (Guido Reni, Velázquez, Murillo, Zurbarán, Tiepolo, etc. ${ }^{\text {II) }}$ s'exercent dans la création de la nouvelle iconographie du dogme, sous le strict contrôle des commissionnaires ecclésiastiques.

II. Parmi les nombreux travaux sur l'iconographie de la Vierge Immaculée, voir Mirella Levi d'Ancona, The Iconography of the Immaculate Conception in the Middle Ages and Early Renaissance, New York, The College Art Association of America, 1957; Émile Mâle, L'art religieux après le Concile di Trente. Étude sur l'iconographie de la fin du XVI siècle, du XVII et du XVIII siècles, Paris, Colin, I932; Louis Réau, Iconographie de l'art chrétien, Paris, PUF, I955. 
La Conception Immaculée, comme l'indique son nom, concerne l'engendrement et représente une manière de légitimer et de normaliser l'union hétérosexuelle. Contrairement à un lieu commun très répandu, il ne concerne pas l'engendrement du Christ mais celui de Marie, sa mère ${ }^{\mathrm{I} 2}$. Pour les franciscains, la Vierge aurait conçu le Christ dans la pureté absolue car par un miracle s'accomplissant lors de son propre engendrement par l'union d'Anne et Joachim, Marie n'aurait pas été contaminée par le pêché originel qui se transmet, selon la théologie chrétienne, par l'acte sexuel des parents. Pure et immaculée avant sa naissance, la Vierge serait devenue cette figure au destin d'exception, ontologiquement différente de ses parents et de toute l'humanité, soumise à l'inévitable corruption du péché originel.

Parmi les représentations de la conception de la Vierge, avant la Réforme, on peut citer la fresque La rencontre à la porte dorée (1304-I306) ${ }^{\mathrm{I3}}$, peinte par Giotto dans la chapelle des Scrovegni à Padoue, sur commande d'Enrico Scrovegni, riche banquier et homme d'affaires. Giotto y représente au premier plan Anna et Joachim en train de s'embrasser. Cette action, qui est la métaphore de l'union hétérosexuelle, est légitimée et sanctifiée comme le montre l'auréole éclairant les visages d'Anne et Joachim. Si Giotto valorise le couple parental à travers une représentation qui comporte l'allusion métaphorique à leur union sexuelle, à partir du Concile de Trente cela ne sera plus possible. L'idéologie du Concile de Trente n'exalte que la chasteté en tant que valeur et établit une doctrine fortement répressive de la sexualité. Toute allusion à la concupiscence sera bannie de l'espace consacré. La vision coupable des unions hétérosexuelles s'approfondit justement au moment où le mariage, qui relevait auparavant de l'autorité civile, devient un sacrement dont l'Église détient le monopole.

En acquérant la gestion juridique et le contrôle sur les mariages, l'Église ne procède pas pour autant à une véritable sacralisation des unions conjugales. Dans la mesure où il comporte l'accès à la sexualité, le mariage met les conjoints en position d'infériorité par rapport aux clercs. Et ceci est vrai même si la sexualité hétérosexuelle est "autorisée» au sein du mariage en fonction de la finalité reproductive. Le mariage demeure un "moindre mal», un remède pour ceux qui ne peuvent pas assumer la chasteté, fondement de la supériorité morale des clercs. La condition de minorité des

I2. Ce quiproquo à l'égard de l'interprétation du dogme dans le sens commun est une conséquence de la construction iconographique de la Vierge Immaculée qui traduit en images une nouvelle élaboration normative de la maternité entièrement soumise au contrôle des ecclésiastiques.

13. La reproduction de la fresque peut être visionnée sur le site <www.wga.hu/support/viewer/z.html> (consulté le 22 octobre 20II). 
laïcs — et la soumission qu'elle implique à l'autorité ecclésiastique - est produite et signifiée à travers une construction normative spécifique des identités familiales selon le genre.

La fonction principale des religions consiste dans l'organisation sociale des rites de passages et dans la définition de l'ordre symbolique où sont exprimés et normalisés les sentiments et les pulsions qui structurent les individus dans leur dimension subjective et intersubjective. Dans le cadre catholique, le symbole de la Conception Immaculée acquiert précisément cette fonction de symbole dominant dans l'organisation du sens subjectif et social du rite matrimonial à partir de sa finalité normative qu'est la reproduction.

C'est en suivant l'évolution de l'iconographie sacrée du dogme que Luisa Accati montre la nouvelle fonction sociale et politique du symbole dans le cadre du renouveau doctrinaire de l'Église de Rome. À partir du $\mathrm{XVI}^{\mathrm{e}}$ siècle, le dogme de l'engendrement de Marie n'est plus représenté par l'image du couple biblique de ses parents, Anne et Joachim, mais par la seule figure de la Vierge. La question de l'engendrement miraculeux et sans péché se résume désormais dans la seule personne de la Vierge, Mère du Christ et de l'Église ${ }^{\mathrm{I} 4}$. Comment et pourquoi se produit-il cet effacement du couple parental qui représentait l'engendrement? Quelle représentation de la conception, et donc de la relation hétérosexuelle, est présentée à travers la figure isolée de la Vierge? Quel sens faut-il donner en particulier à la disparition de la figure masculine et paternelle dans la représentation de la conception?

Dans une fresque de Tiepolo, l'un des artistes majeurs du thème ${ }^{15}$, la Vierge Immaculée apparaît comme une jeune fille, très belle, qui d'un regard altier tourné vers le bas domine toute seule le tableau. Au-dessus de sa tête, la colombe blanche représente le Saint-Esprit. Appuyée sur un globe, symbole de la sphère temporelle, elle est habillée en blanc, la couleur de la pureté, symbolisée aussi par la fleur de lys qui se trouve entre les mains d'un ange à ses pieds. Médiatrice entre l'ici-bas et l'au-delà, la Vierge est enveloppée dans un manteau bleu signalant son appartenance au Ciel. L'unique allusion à la concupiscence, c'est-à-dire à la relation hétérosexuelle entre les parents, est représentée par le serpent qui mord

I4. Pour l'évolution de la doctrine du dogme et sa signification dans l'imaginaire socio-politique, voir Luisa Accati, «La bella e l’immaginazione», dans Il mostro e la bella, ouvr. cité, p. 57-80.

I5. Pour une reproduction de la fresque Conception Immaculée (I767-I769) peinte par Giovanni Battista Tiepolo, voir le site : <www.museodelprado.es/it/visita-il-museo/I5-opere-maestre/ficha-de-obra/zoom/I/obra/ immacolata-concezione/oimg/o/> (consulté le Io février 20I2). 
une pomme et que la Vierge écrase sous ses pieds, signifiant son triomphe sur le péchér ${ }^{16}$.

Une fois éliminé le couple hétérosexuel, dans la représentation de l'engendrement miraculeux il ne reste plus que la silhouette de Marie, la mère par excellence. Marie est le "vase d'élection", le ventre ou contenant pur, parfait, accueillant passivement la volonté divine. Elle est tellement pure et parfaite qu'elle devient un corps désincarné, montré toujours dans un état de suspension entre ciel et terre. Corps passif, la Vierge est un sujet sans volonté, sans déterminations individuelles, qui accueille passivement la maternité du Christ, décidée par Dieu depuis l'éternité.

Dans cette idéalisation de la maternité et de l'engendrement la présence de toute figure masculine paternelle devient inutile. Au temps de Giotto, la dimension sainte de la Mère Vierge était encore définie en relation à la sphère humaine et la représentation de la conception impliquait la figuration du couple de parents. Le couple parental représentait la médiation entre la volonté divine et la naissance de la progéniture divine. Avec la Contre-Réforme, l'élévation mystique de la Vierge Immaculée transforme la maternité en pure métaphore qui n'a plus rien de commun avec l'engendrement humain hétérosexuel. L'exclusion de toute figure masculine renforce ce travail d'abstraction de la maternité qui apparaît totalement déliée de la relation hétérosexuelle, confirmant ainsi la dévalorisation radicale de celle-ci.

La Vierge demeure un modèle normatif pour les femmes, un modèle qui ne prend en compte que la dimension maternelle. De plus, c'est un modèle d'identification très pénalisant, car il suppose la passivité et l'absence de volonté des femmes qui "subissent» la maternité comme douleur nécessaire en vue du salut. Cette vision catholique de la maternité implique aussi la dévotion absolue aux enfants jusqu'à l'effacement de soi. Mais surtout le modèle de maternité de la Vierge impose la subordination des épouses et mères à l'autorité ecclésiastique. Si la soumission au mari est requise, à la différence du patriarcat protestant, en terre catholique l'assujettissement aux clercs est bien plus important. Et toute la symbolique que l'on vient de décrire, marquée par la forclusion de la figure paternelle, est précisément conçue pour réaffirmer la prééminence de l'autorité des ecclésiastiques sur l'autorité des hommes laïcs ${ }^{17}$.

16. Pour une analyse détaillée des éléments symboliques contenus dans l'iconographie de la Vierge Immaculée et de leur signification, voir Luisa Accati, «La madre come sogno del figlio», dans Il mostro e la bella, ouvr. cité, p. 193-202.

17. Pour une analyse des enjeux et des conséquences de l'élimination de la figure de l'époux et du père de l'imaginaire catholique, voir en particulier le chapitre «I figli e la doppia esclusione del mostro", dans ibid., p. II5-202. 
À travers l'élaboration idéologique de la maternité de la Vierge, on définit un dispositif de normalisation des identités genrées qui permet d'articuler les relations d'autorité entre les "pères" laïcs et les "fils» clercs par la médiation des mères. Le contrôle sur la symbolique de l'engendrement - et par là sur la représentation des unions hétérosexuelles - est en effet organisé à partir du contrôle du corps des femmes et de leur soumission aux ecclésiastiques. L'exclusion de la figure paternelle masculine de l'iconographie sert en effet à dévaloriser et à forclore le sujet antagoniste de l'autorité cléricale. Si l'homme laïc et père est exclu de la représentation, le clerc y est intégré de manière métaphorique. Car en effet l'image de la Vierge renvoie à l'institution sacrée et à l'ensemble des ecclésiastiques. Mère de l'Église, la Vierge est ce «vase d'élection» maternel pur et parfait qui contient les clercs en tant que sujet collectif. Elle est la figure d'autorité à laquelle s'identifient les successeurs du Christ. Si la Vierge devient une figure symbolique d'identification pour des hommes, c'est qu'en effet sa dimension maternelle a pu être appropriée par les clercs qui ont fait de l'expérience de la dépendance filiale de la mère une métaphore de la relation d'autorité.

Le sens et les conséquences de cette identification paradoxale des clercs avec le symbole de la maternité, qui par ailleurs caractérise toute la culture monastique chrétienne, sont analysés par Luisa Accati au prisme de la théorie psychanalytique freudienne. Sur le plan subjectif, l'identification du fils à la mère peut être interprétée comme une projection fantasmatique d'un adulte demeurant prisonnier d'un lien fusionnel avec la mère. D'une part, l'idéalisation de la Mère Vierge depuis l'éternité, donc à jamais inviolable, représente une protection contre le risque de fantasmes incestueux. D'autre part, par cette identification à la mère, le fils peut attribuer à lui-même cette forme de pouvoir absolu détenu par la mère dans sa relation avec le petit enfant. Dans le fantasme masculin clérical, la dépendance du petit enfant de sa mère se transforme en pouvoir à exercer sur la partie féminine du corps politique et tout particulièrement sur les femmes destinées au mariage et donc à la maternité. Le dispositif normatif de la Conception Immaculée sert à légitimer l'autorité de l'Église sur le corps politique à partir du contrôle des femmes et de la normalisation des identités féminines. Luisa Accati montre que ce dispositif normatif, basé sur la dévalorisation radicale du couple hétérosexuel et sur l'idéalisation de la maternité, est finalisé au contrôle de la famille laïque et à l'affermissement de l'autorité «maternelle» de l'Église et des ecclésiastiques. 


\section{La Mujer Barbuda de José Ribera ou le paradoxe du corps maternel masculin}

L'icône de la Vierge Immaculée représente un dispositif spécifique de pouvoir produisant des identités genrées qui ne sont pas fondées sur le sexe naturel, comme on pourrait le croire en suivant les analyses de Judith Butler sur le patriarcat. L'identification des clercs avec le symbole de la Mère montre la souplesse qui caractérise le contexte normatif catholique à l'égard de la construction du genre en relation au sexe naturel. De même dans le patriarcat catholique on ne retrouve pas une valorisation de l'hétérosexualité comparable à celle des cultures protestantes. Dans les cultures catholiques, on réaffirme la supériorité de ceux qui (les clercs) s'abstiennent de toute sexualité.

Pour illustrer les ambiguïtés de l'identité genrée dans les cultures catholiques, Luisa Accati cite en exemple un tableau peint en I63I par l'artiste espagnol José Ribera, auteur par ailleurs d'une Vierge Immaculée et expert donc du dogme pour avoir eu à suivre les strictes consignes des ecclésiastiques concernant la représentation de la Vierge. Réalisée sur commande du duc d'Alcalé, La Mujer Barbuda ou Magdalena Ventura con su esposo $e$ hijo $^{18}$ illustre l'étrange histoire et le miracle d'une pauvre femme du royaume de Naples qui, à l'âge de 37 ans, commence à avoir une barbe épaisse et, quinze ans après, accouche d'un enfant.

Thème à la mode dans la culture espagnole du Siècle d'or, la femme à barbe de Ribera se démarque par son originalité. À la différence d'autres représentations classiques, Ribera ne montre pas la femme barbue toute seule, mais il la met en scène avec sa famille ${ }^{\text {19 }}$. Au milieu du tableau se trouve la figure imposante de la mère avec sa barbe bien fournie, en train d'allaiter son bébé. Un gros sein surréaliste, placé au milieu du buste, sort du haut de sa robe qui est en effet une parure d'ecclésiastique. Derrière cette mère-prêtre se trouve le mari. C'est un vieux monsieur, presque caché dans la pénombre. Le mari et Magdalena ont presque les mêmes mains, les mêmes yeux et les deux visages se ressemblent au point que l'on pourrait croire que le mari est la version vieillie de Magdalena. Leurs regards inquiets et étonnés percent la représentation et adressent au spectateur du tableau une interrogation, une stupeur irrésolue, un étonnement si fort

I8. Pour la reproduction du tableau, voir <http://es.fundacionmedinaceli.org/coleccion/fichaobra.aspx? id=378> (consulté le Io février 20I2).

19. Sur le tableau de Ribera dans le cadre de l'iconographie du thème de la femme à barbe en Espagne, voir Fernando R. de la Flor, "José de Ribera: la proyeccíon final del tema de la barbuda", dans La península metafisica. Arte, literatura y pensamiento en la España de la Contrarreforma, Madrid, Biblioteca Nueva, 1999, p. 300-304. 
qui ne semble pas traduire simplement la merveille face au miracle de la naissance prodigieuse. En effet, comme le relève Luisa Accati, la famille miraculée n'est pas une famille hétérosexuelle. Le tableau semble être une sorte de version profane de l'étonnante construction de la maternité symbolique de la Vierge Immaculée. «Ribera aperçoit clairement la dimension paradoxale de la relation entre la réalité et le symbole, entre la mère réelle et la Vierge, mère et symbole de l'Église, entre la famille naturelle et la famille théologique ${ }^{20}$." Comme le précise Luisa Accati, Ribera montre l'étrangeté de ce passage "du genre de la mère, qui ne peut qu'être de sexe féminin dans le réel " à la symbolique catholique "où le sexe de la mère est masculin ${ }^{21} »$. Le tableau fait aussi apparaitre explicitement un autre élément qui demeure forclos dans la représentation de la Vierge Immaculée : la confrontation entre la figure filiale ecclésiastique et la figure paternelle laïque. Le symbole de l'Immaculée sert précisément à gérer la relation entre ces deux classes d'hommes et à produire discrètement l'effacement de l'une des deux classes - celle des laïcs. Le tableau de Ribera, en représentant le père, montre la tension que l'icône de la Vierge devrait résoudre et fait ainsi apparaître toute l'ambiguiité de la construction de l'autorité maternelle des clercs. Il montre ainsi les difficultés ou les réserves à l'accepter au moment où commence la véritable diffusion du dogme dans l'iconographie.

Selon Luisa Accati, le couple du tableau de Ribera illustre l'hypothèse selon laquelle la symbolique catholique n'exclue pas un imaginaire homosexuel. Au contraire, elle en produit un, lié à la construction du pouvoir «maternel» ecclésiastique ${ }^{22}$. Dans la performance picturale, l'ambiguïté du corps de Magdalena, mère cléricale et masculine, montre bien que dans l'idéologie catholique les identités genrées ne sont pas construites sur la base de la référence au «sexe naturel».

L'étonnement de Magdalena peut être interprété comme une invitation à s'interroger sur l'étrangeté qu'impose le dispositif normatif ecclésiastique du pouvoir. Si le tableau permet de saisir à ses débuts le dispositif normatif catholique de la différence des sexes dans sa spécificité, c'est en faisant l'histoire de cet ordre symbolique — en le différenciant de celui des autres confessions chrétiennes - que l'on peut véritablement assurer

20. Luisa Accati, «Il marito respinto e il padre assente, ovvero il conflitto con la madre da Giotto a Buńuel», dans Saveria Chemotti, Madre de-genere: la maternità tra scelta, desiderio e destino, Padoue, Il Poligrafo editore, 2009, p. 239 (notre traduction).

2I. Ibid.

22. Pour un approfondissement concernant la dimension implicitement homosexuelle de l'imaginaire catholique du pouvoir, voir Luisa Accati, Il mostro e la bella, ouvr. cité, p. 154-I57. 
le démontage des normes qui font fonctionner le patriarcat catholique. Ainsi, la prise en compte de l'histoire spécifique des dispositifs de pouvoir produisant la signification normative des genres nous semble être le chemin principal pour définir les voies d'une véritable émancipation des individus. 